\section{In the works}

CONVENTIONAL thinking comes under fire with a report by $M$. Carmo-Fonseca and colleagues which provides evidence for the existence of RNA-processing 'factories' in the nuclei of eukaryotic cells (EMBO J. 10, 195-206; 1990). It has long been thought that the small nuclear ribonucleoproteins (snRNPs) involved in the splicing of pre-messenger RNA are distributed throughout the nucleus. Not so, say Carmo-Fonseca et al., who using antibodies against snRNP-specific proteins and snRNA antisense probes show that these snRNPs are found mainly at a few distinct sites. The implication is that eukaryotic nuclei contain an organelle that either assembles mature snRNPs or uses them in pre-mRNA splicing.

\section{Something for nothing}

If not quite routine, the capture and manipulation of atoms in beams of laser light is nonetheless becoming popular in optics laboratories. But ghostly as these optical forces may seem, they are quite tangible compared with one created from nothing now proposed by $S$. Haroche et al. and by B.-G. Englert et al. (Europhys. Lett. 14, 19-24 \& 25-31; 1991). The idea exploits the omnipresent electromagnetic fluctuations that characterize the vacuum. In a micromaser, a superconducting cavity selects a single frequency that resonates with the microwave radiation from an excited atom. The ghostly vacuum fluctuation quickly stimulates the atom to radiate. The force now discovered arises if the atomic and cavity frequencies are not quite matched. As the atom tries to radiate into the wrong microwave mode, it is either drawn into the cavity's centre or repelled from it, depending on which frequency is higher.

\section{Bull hit}

THE high affinity of the protein, avidin, for its natural ligand, biotin, has given rise to a range of biochemical fancies, the latest of which offers a means of speeding an enzymic bullet to a selected target (E. A. Bayer et al. Biochemistry 29, $11274-11279 ; 1990)$. Avidin is a

tetramer, so when it takes up one molecule of a biotinylated protease, three biotin sites remain vacant. If one of these is allowed to fasten itself onto a biotinylated substrate, proximity ensures rapid attack by the protease, which shows no interest at all in other substrate molecules in the solution. So a working biotinylated enzyme can be brought to a standstill by simply adding avidin. Another way to present the substrate to the enzyme is by way of a biotinylated anti-substrate antibody. The authors envisage applications of their invention to substrates in complex systems selected constituents of cell surfaces are one example.

temperatures type I PSCs should be present, and the ozone mixing ratio at the same altitude. For these trajectories, however, the amount of sunlight received by the parcels was relatively constant.

This result raises the important question of which factors control polar ozone depletion. The catalytic cycles believed to cause ozone destruction all require sunlight to operate, which is thus a prerequisite. Given this, a key question is the extent to which air can be processed by a cold region containing PSCs and maintain the conditions of perturbed chemistry. In other words, what is the timescale for the deactivation of chlorine after an air parcel has passed through such a cold region? These results suggest that the amount of time air is exposed to PSCs is the most important factor in determining the ozone depletion. The amount of denitrification will depend on this length of time, and CONSERVATION BIOLOGY

\title{
Overgrazing overstated
}

\section{Ruth Mace}

SOMETIMES we are so sure of something that we don't need to see the evidence. That Africa's rangelands are being reduced to desert through overgrazing by domestic livestock is received wisdom. But, as became plain at a recent meeting*, such a view may be seriously flawed.

Conventional thinking is based on two premises. Simple predator-prey models of herbivory illustrate how high grazing pressure can hold a system below its maximum productivity'. And Hardin's classic paper ${ }^{2}$ on the intrinsic propensity of individuals to overuse resources owned in common explains why herders are likely to push communal rangeland into this overgrazed state. For decades, most pastoral development projects in Africa have been based on the principle that selfinterest makes pastoralism underproductive and environmentally damaging. So there has been an emphasis on communal projects, such as group ranches, grazing rotation schemes and reduced herd sizes, on the grounds that short-term inconvenience to herders would act for the greater good. The problem is that these programmes do not work, and generally end in the ignominious retreat of the development agencies.

Words such as 'overgrazing' and 'overstocking' assume that we know what the right stocking density is, which is taken to be when a particular grazing system is at or below its 'carrying capacity'. But great uncertainty centres on the concept of carrying capacity ${ }^{i-4}$. Population biologists recognize it as the population size at which a population stops growing $(K)$. Range

* Savannah Development and Pasture Production. Woburn UK, 19-21 November 1990. thus so will the rate of chlorine deactivation as $\mathrm{ClO}$ is converted to $\mathrm{ClONO}_{2}$ by reaction with $\mathrm{NO}_{2}$. Thus, it is not enough for air merely to have encountered PSCs and the total time spent at cold temperatures is important.

Alternatively ${ }^{13}$, the temperature of an air parcel may control the amount of ozone destruction via the thermal decomposition of $\mathrm{Cl}_{2} \mathrm{O}_{2}$. In warmer conditions, for which this thermal decomposition becomes significant relative to the photolysis of $\mathrm{Cl}_{2} \mathrm{O}_{2}$, ozone destruction will effectively stop. As Hofmann and Deshler point out, studying the new data with a detailed photochemical trajectory model will help to elucidate the mechanisms controlling ozone loss.

Martyn Chipperfield is at ERAM, Centre National de Recherches Météorologiques, 42 Avenue Coriolis, 31057 Toulouse, France. scientists, who have generally assumed that environmental damage occurs when livestock numbers exceed carrying capacity, have borrowed the term and given it a different meaning, or rather a range of meanings, which are more akin to an optimal stocking density rather than a maximum (nearer $K / 2$ rather than $K$ if a population were to grow logistically).

Compounding the confusion is the way range carrying capacity is usually calculated - by estimating the total edible plant biomass produced annually, multiplying it by a 'proper use factor' and dividing it by the amount of forage an animal needs to survive, thereby giving the theoretical number of animals that a range can sustain. All of these measures suffer from variability, error and subjectivity (B. Norton, Utah State University), giving a final figure that it is probably not too cynical to describe as arbitrary. Plant productivity is strongly dependent on rainfall, which varies greatly from one year to the next. The 'proper use factor' is rarely more than a rule of thumb, as the relationship between how much forage is left uneaten and next year's growth is not well understood. Even estimation of the quantity of forage needed by an animal is not straightforward, as it will depend on the economic objectives of the herder. For example, five thin animals may be better than three fat ones if milk and hides are the most important products, which is commonly the case in traditional systems.

Norton illustrated how unreliable this method can be with an example where livestock numbers in Somalia were stated to habitually exceed eightfold the care- 